\title{
Understanding the impact of physical activity in COPD outcomes: moving forward
}

\author{
Richard ZuWallack ${ }^{1}$ and Cristóbal Esteban²
}

\begin{abstract}
Affiliations: ${ }^{1}$ Pulmonary and Critical Care, St Francis Hospital, Hartford, CT, USA. ${ }^{2}$ Pneumology Dept and Red de Investigación en Servicios Sanitarios y Enfermedades Crónicas, Hospital Galdakao-Usansolo, Galdakao, Spain.
\end{abstract}

Correspondence: Richard ZuWallack, St Francis Hospital, Pulmonary and Critical Care, 114 Woodland, Hartford, CT 06105, USA. E-mail: rzuwalladstfranciscare.org

-

@ERSpublications

From observational studies, it is now clear that physical inactivity is linked with poor outcome in COPD http://ow.ly/AJ7mF

Arguably, three outcome categories are of greatest importance to chronic obstructive pulmonary disease (COPD) patients, their families and their caregivers: health-related quality of life (including symptom burden); hospitalisations and other forms of healthcare utilisation; and, of course, mortality. Lower levels of baseline physical activity are not only prevalent in patients with COPD [1,2], they are associated a negative impact in each of these three outcome categories [3-5].

Of considerable importance to healthcare professionals, increases in physical activity appear to predict enhanced health-related quality of life and reduced hospitalisations. For example, in an analysis of 391 patients with COPD who had physical activity and health-related quality of life assessments at baseline and 5 years later [6], those who increased or maintained their high levels of physical activity had clinically meaningful improvements in quality of life. In contrast, those who stayed at a low level or decreased their physical activity had a worsening in this outcome. From one perspective, this comes close to a tautology, as physical function impairment and distressing symptoms associated with physical activity are prominent components of health-related quality of life questionnaires. With respect to healthcare utilisation, a longitudinal analysis of COPD patients over 5 years demonstrated that the change in physical activity over the first 2 years significantly predicted hospitalisations 3 years later: those without regular physical activity, those who maintained a low level of physical activity or those who decreased their physical activity were at significantly greater risk of being hospitalised than those with higher levels of physical activity [7].

The reanalysis of the very large Copenhagen City Heart Study database by VAES et al. [8], reported in this issue of the European Respiratory Journal, adds new and important information by relating changes in physical activity to mortality risk in COPD. We already know there are often strong associations between low levels of physical and mortality. For instance, all-cause mortality risk is higher in patients with coronary artery disease and cancer who have lower levels of physical activity $[9,10]$. This also holds true for COPD: using Copenhagen City Heart Study data, GARCIA-AYMERICH et al. [3] demonstrated that, in participants meeting spirometric criteria for COPD, those with very low levels of self-reported physical activity had a higher risk of hospitalisation and mortality over approximately the next 17 years than those who participated in regular physical activity. This negative relationship between physical activity and poor outcome remained in their model even after controlling for potential relevant confounding factors. This study provides strong evidence that physical inactivity is bad for individuals with COPD. Subsequent studies by WASCHKI et al. [4] and GARCIA-RIO et al. [11] demonstrated that directly measured physical activity was a potent predictor of all-cause mortality in COPD patients. The latter actually demonstrated a linear dose-response relationship between level of physical activity and mortality.

Received: Aug 182014 | Accepted: Aug 192014

Conflict of interest: Disclosures can be found alongside the online version of this article at erj.ersjournals.com

Copyright @ERS 2014 
VAES et al. [8] go one step further (no pun intended) in linking physical activity to outcome, by presenting strong evidence relating changes in physical activity to mortality risk in COPD. Patients with moderate or high self-reported physical activity at baseline who subsequently decreased to low physical activity at followup were at particularly high risk of dying over the ensuing 17 years (adjusted hazard ratios for death 1.73 and 2.35 , respectively). The obvious inference from this analysis is that becoming physically inactive reduces the likelihood of a prolonged earthly existence.

A good scientific study often raises more questions than it answers. With the study by VAES et al. [8], two come to mind. 1) Why was the increase in physical activity in those patients with a low level at baseline not translated into a decreased mortality risk? 2) Is it really the lower level physical activity that is factor driving poor outcome, or is it simply an epiphenomenon correlated with other, causal variables?

Perhaps most disturbing for those caring for patients with advanced COPD, in whom severe physical inactivity is so prevalent, is the observation that an increase in physical activity from a low baseline level did not confer survival benefit. The investigators suggest a rather literal interpretation of the data: that there may be a threshold below which increasing activity simply cannot improve mortality risk. In essence, it would be better not to fall into the abyss of physical inactivity rather than to try to climb out of it. In contrast to this discouraging observation, through avoiding activity stratification and using a simulation model, MinTon et al. [12] demonstrated just the opposite effect: individuals (not necessarily COPD patients) who are least active are most likely to gain a mortality benefit through modestly increasing activity. Indeed, this purported beneficial effect in hypothetical sedentary individuals was greater than in those who were already moderately or highly active. Of course, whether this simulation model holds up for real patients with COPD remains to be demonstrated.

Analyses of changes in physical activity in other diseases point to the beneficial effects of relatively small increases in physical activity on variables that influence mortality and mortality risk. For instance, in overweight-to-obese adults, a modest increase in physical activity was associated with reduced arterial stiffness, a risk factor of future cardiovascular events [13]. Furthermore, in patients with a diagnosed cardiovascular disease, an inverse association between physical activity and mortality was shown (i.e. secondary prevention) over an average of 5.6 years in follow-up [14].

Perhaps COPD patients with a very low level of baseline physical activity cannot improve their bad prognosis by pulling themselves up by their bootstraps to reach the intensity levels necessary for a change in mortality outcome. An established and intensive pulmonary rehabilitation programme might help in this regard. For example, in patients with post-infarction heart failure, high-intensity training reverses left ventricular remodelling, and improves endothelial function and aerobic capacity [15]. Further knowledge in this area would be of keen interest to pulmonary rehabilitation professionals, who often treat very inactive COPD patients with the expectation that, in addition to symptom relief and functional status improvement, some gain in healthcare utilisation and mortality risk may result from the comprehensive intervention.

A second issue raised by the VAES et al. [8] study and other observational studies is the presumed attribution of causality to physical activity. Is physical (in)activity really the culprit driving mortality or is it just along for the ride? Without question, the large body of evidence from correlational studies in COPD, other diseases and healthy individuals does point to a causal connection between inactivity and mortality. However, to use an extreme example, a chronically ill, multimorbid, bedridden patient with almost no physical activity would be expected to have a high risk of dying, but that risk is probably mainly due to the morbidity, not the physical inactivity per se.

There are probably already enough observational studies linking physical inactivity with poor outcome in COPD. What we really need are prospective, randomised clinical trials testing whether a change in physical activity resulting from an intervention has a demonstrable effect on subsequent outcome. This would be no small undertaking, as it is not particularly easy to increase physical activity in COPD [16, 17], and studies like this would require considerable resources and time to complete. However, the information gained would be of considerable importance in directing increasingly scarce medical resources toward realisable goals.

\section{References}

1 Pitta F, Troosters T, Spruit MA, et al. Characteristics of physical activities in daily life in chronic obstructive pulmonary disease. Am J Respir Crit Care Med 2005; 171: 972-977.

Watz H, Waschki B, Meyer T, et al. Physical activity in patients with COPD. Eur Respir J 2009; 33: 262-272.

Garcia-Aymerich J, Lange P, Benet M, et al. Regular physical activity reduces hospital admission and mortality in chronic obstructive pulmonary disease: a population based cohort study. Thorax 2006; 61: 772-778.

4 Waschki B, Kirsten A, Holz O, et al. Physical activity is the strongest predictor of all-cause mortality in patients with COPD: a prospective cohort study. Chest 2011; 140: 331-342.

5 Zanoria SJ, ZuWallack R. Directly measured physical activity as a predictor of hospitalizations in patients with chronic obstructive pulmonary disease. Chron Respir Dis 2013; 10: 207-213. 
Esteban C, Quintana JM, Aburto M, et al. Impact of changes in physical activity on health-related quality of life among patients with COPD. Eur Respir J 2010; 36: 292-300.

7 Esteban C, Arostegui I, Aburto M, et al. Influence of changes in physical activity on frequency of hospitalization in chronic obstructive pulmonary disease. Respirology 2014; 19: 330-338.

8 Vaes AW, Garcia-Aymerich J, Marott JL, et al. Changes in physical activity and all-cause mortality in COPD. Eur Respir J 2014; 44: 1199-1209.

9 Schnohr P, Lange P, Scharling H, et al. Long-term physical activity in leisure time and mortality from coronary heart disease, stroke, respiratory diseases, and cancer. The Copenhagen City Heart Study. Eur J Cardiovasc Prev Rehabil 2006; 13: 173-179.

10 Andersen LB, Schnohr P, Schroll M, et al. All-cause mortality associated with physical activity during leisure time, work, sports, and cycling to work. Arch Int Med 2000; 160: 1621-1628.

11 Garcia-Rio F, Rojo B, Casitas R, et al. Prognostic value of the objective measurement of daily physical activity in COPD patients. Chest 2012; 142: 338-346.

12 Minton J, Dimairo M, Everson-Hock E, et al. Exploring the relationship between baseline physical activity levels and mortality reduction associated with increases in physical activity: a modelling study. BMJ Open 2013; 3: e003509.

13 Hawkins M, Gabriel KP, Cooper J, et al. The impact of change in physical activity on change in arterial stiffness in overweight or obese sedentary young adults. Vasc Med 2014; 19: 257-263.

14 Hamer M, Stamatakis E. Physical activity and mortality in men and women with diagnosed cardiovascular disease. Eur J Cardiovasc Prev Rehabil 2009; 16: 156-160.

15 Wisløff U, Støylen A, Loennechen JP, et al. Superior cardiovascular effect of aerobic interval training versus moderate continouos training in heart failure: a randomized study. Circulation 2007; 115: 3086-3094.

$16 \mathrm{Ng}$ LW, Mackney J, Jenkins S, et al. Does exercise training change physical activity in people with COPD? A systematic review and meta-analysis. Chron Respir Dis 2012; 9: 17-26.

17 Casaburi R. Activity promotion: a paradigm shift for chronic obstructive pulmonary disease therapeutics. Proc Am Thorac Soc 2011; 8: 334-337. 